\title{
6 SATISFAÇÃO E SOBRECARGA DE TRABALHO ENTRE TÉCNICOS DE ENFERMAGEM DE HOSPITAIS PSIQUIÁTRICOS
}

\author{
| Gisele de Lacerda Chaves Vieira ${ }^{1}$
}

\section{RESUMO}

CONTEXTO: As situações vivenciadas pelos profissionais de enfermagem em instituições psiquiátricas podem ser geradoras de sobrecarga e insatisfação relacionadas ao trabalho. Essas, por sua vez, interferem na qualidade dos serviços prestados à população.

OBJETIVOS: Caracterizar o perfil sociodemográfico dos técnicos de enfermagem que trabalham em hospitais psiquiátricos, identificar os níveis de satisfação e sobrecarga relacionados ao trabalho e verificar a relação entre esses níveis.

MÉTODOS: Estudo transversal realizado com 103 técnicos de enfermagem de dois hospitais psiquiátricos. Para a coleta de dados, foi utilizado o questionário sociodemográfico e ocupacional e as escalas SATIS-BR e IMPACTO-BR. Foram calculadas médias, percentis e frequências. A relação entre as duas escalas foi verificada por meio do coeficiente de correlação de Spearman.

RESULTADOS: Foi registrado escore médio global de 3.1 para a escala de satisfação e de 2.2 para a sobrecarga relacionada ao trabalho. Os menores níveis de satisfação estiveram relacionados às subescalas oportunidade de participação no serviço (2.8) e condições de trabalho (2.7). Já para a sobrecarga de trabalho, o maior escore foi encontrado para as repercussões sobre o funcionamento da equipe (2.5). A sobrecarga relacionada ao trabalho e a satisfação dos profissionais apresentou relação estatística significante entre a maioria das dimensões avaliadas.

CONCLUSÃO: Considerando a influência da sobrecarga do trabalho e dos níveis de satisfação dos profissionais sobre os processos de adoecimento, destaca-se a importância de melhorar as condições de trabalho dos técnicos de enfermagem.

\section{PALAVRAS-CHAVE: Enfermagem psiquiátrica; Saúde do trabalhador; Hospitais psiquiátricos; Satisfação no emprego}

\section{RESUMEN}

"La satisfacción y la sobrecarga de trabajo entre los técnicos de enfermería de hospitales psiquiátricos"

CONTEXTO: Situaciones experimentadas por los técnicos de enfermería en las instituciones psiquiátricas pueden producir sobrecargas y la insatisfacción relacionada con el trabajo. Estos, a su vez, afectan a la calidad de los servicios prestados a la población.

OBJECTIVOS: Caracterizar el perfil sociodemográfico de técnicos de enfermería de hospitales psiquiátricos, identificar los niveles de satisfacción y la carga relacionada con el trabajo y verificar la relación entre estas escalas.

METODOS: Estudio transversal con 103 técnicos de enfermería de dos hospitales psiquiátricos. Para la recolección de datos se utilizó el cuestionario sociodemográfico y laboral y las escalas SATIS-BR y IMPACTO-BR. Se calcularon promedios, percentiles y frecuencias. La relación entre las dos escalas mediante el coeficiente de correlación de Spearman.

RESULTADOS: Se registró la puntuación media global de 3,1 para la escala de satisfacción y 2.2 para el trabajo relacionado con sobrecarga. Los niveles más bajos de satisfacción se relacionan con la oportunidad de participar en el servicio (2.8) y las condiciones de trabajo (2.7). En cuanto a la carga de trabajo se encontró que la puntuación más alta de las repercusiones en el funcionamiento del equipo $(2,5)$. La sobrecarga relacionada con el trabajo y la satisfacción de los profesionales mostró una relación estadísticamente significativa entre la mayoría de las dimensiones evaluadas.

CONCLUSIÓN: Teniendo en cuenta la influencia de la sobrecarga de trabajo y los niveles de satisfacción profesional en procesos de enfermedad pone de relieve la importancia de mejorar las condiciones de trabajo de los técnicos de enfermería.

DESCRIPTORES: Enfermería psiquiátrica; Salud laboral; Hospitales psiquiátricos; Satisfacción en el trabajo

\section{ABSTRACT \\ "Satisfaction and workload among nursing technicians in psy- chiatric hospitals"}

BACKGROUND: Situations experienced by nursing technicians in psychiatric institutions can generate overload and dissatisfaction related to work. These, in turn, affect the quality of services provided to the population.

AIMS: To characterize the sociodemographic profile of nursing technicians who work in psychiatric hospitals, identify levels of satisfaction and burden related to work and the relation between these scales.

METHODS: Cross-sectional study with 103 nursing technicians of two psychiatric hospitals. For data collection was used sociodemographic and occupational questionnaire and the SATIS-BR and IMPACTO-BR scales. Averages, percentiles and frequencies were calculated. The relationship between the two scales was verified using the Spearman correlation coefficient.

RESULTS: Was recorded global mean score of 3.1 for satisfaction scale and 2.2 for overhead work-related. The lowest levels of satisfaction were related to the opportunity to participate in the service (2.8) and working conditions (2.7). As for the workload, the highest score was found for the repercussions on the functioning of the team (2.5). Overloading related to work and the satisfaction of professionals showed a statistically significant relationship between most dimensions evaluated.

CONCLUSION: Considering the influence of the overload of work and professional satisfaction levels on disease processes highlights the importance of improving the working conditions of nursing technicians.

\section{KEYWORDS: Psychiatric nursing; Occupational health; Hospi- tals, psychiatric; Job satisfaction}

Submetido em 17-06-2016

Aceite em 30-03-2017

1 Doutoranda do Programa de Pós-Graduação em Enfermagem da Escola de Enfermagem da Universidade Federal de Minas Gerais - UFMG. Enfermeira na Fundação Hospitalar do Estado de Minas Gerais. Belo Horizonte-MG - Brasil, giselelacerdavi@gmail.com 


\section{INTRODUÇÃO}

Os profissionais de enfermagem representam grande parte dos trabalhadores da área da saúde em instituições hospitalares. Esses profissionais passam grandes períodos no ambiente de trabalho vivenciando situações estressantes que, muitas vezes, são responsáveis pelo seu adoecimento físico e psíquico (Felli, 2012).

A sobrecarga no emprego constitui-se um dos fatores que influencia a qualidade de vida no trabalho que, por sua vez, apresenta relação com os níveis de satisfação associados ao serviço. Ademais, estudos sugerem que os níveis de sobrecarga no emprego e de satisfação são capazes de predizer a qualidade dos serviços prestados aos pacientes em unidades de saúde mental (Rebouças, Legay e Abelha, 2007; Bogaert et al., 2013). Desta maneira, a Organização Mundial de Saúde propõe que a satisfação dos profissionais e a sobrecarga no trabalho sejam também avaliadas como indicadores de qualidade desses serviços (Organização Mundial de Saúde [OMS], 2001).

Em hospitais psiquiátricos, especialmente, os técnicos de enfermagem vivenciam de forma constante um clima de tensão emocional e de frustração, uma vez que prestam assistência a pacientes crônicos e portadores de transtornos mentais graves que apresentam poucas mudanças ao longo dos anos. Aliado a isso, esses profissionais geralmente enfrentam duplas jornadas de trabalho, baixos salários e condições de trabalho muitas vezes precárias que geram insatisfação no trabalho, sobrecarga física e psíquica (Leal, Bandeira e Azevedo, 2012).

Apesar disso, ainda existem poucas pesquisas nacionais e internacionais que visem identificar a sobrecarga no trabalho e os níveis de satisfação dos profissionais de enfermagem que trabalham em hospitais psiquiátricos (Hamaideh, 2011; Leal et al., 2012; Ferreira, 2015; Alves et al., 2013; Gandhi, Sangeetha, Ahmed \& Chaturvedi, 2014). No Brasil, estudos que utilizaram escalas validadas encontraram maiores níveis de sobrecarga no trabalho relacionados aos aspectos emocionais e menores níveis de satisfação relacionados às condições de trabalho (Leal et al., 2012; Ferreira, 2015; Alves et al., 2013). Porém, é importante destacar que as análises realizadas nesses estudos não consideraram as diferentes categorias profissionais, não sendo possível, então, identificar a sobrecarga e os níveis de satisfação entre os profissionais da equipe de enfermagem.
Considerando que os técnicos de enfermagem são os integrantes da equipe de saúde mental que passam maiores períodos em contato com os pacientes internados em hospitais psiquiátricos, constatou-se a necessidade de identificar os níveis de satisfação e de sobrecarga relacionados ao trabalho, pondo em destaque essa categoria profissional. Além disso, buscou-se verificar a relação entre os níveis de satisfação encontrados e os escores obtidos para a sobrecarga relacionada ao trabalho.

\section{MÉTODOS}

Trata-se de estudo transversal realizado, em dezembro de 2014, em dois hospitais psiquiátricos do estado de Minas Gerais - Brasil. As instituições somam 253 leitos e 255 profissionais de enfermagem de nível médio. Esses hospitais oferecem serviços de atendimento ambulatorial, urgência e internação hospitalar.

Todos os profissionais de enfermagem de nível médio foram convidados a participar da pesquisa. Os critérios de inclusão foram: atuar como técnico ou auxiliar de enfermagem no hospital e prestar assistência direta aos pacientes. Os profissionais que não participaram do estudo não apresentaram diferenças em relação às variáveis sexo, idade e tempo de vínculo com o serviço. Os motivos de não participação foram devidos à ocorrência de licenças, férias, falta de retorno dos instrumentos de pesquisa ou recusa à participação no estudo. Os profissionais foram convidados a responder três questionários: o questionário sociodemográfico e ocupacional, a Escala SATIS-BR e a Escala IMPACTO-BR. Essas escalas foram elaboradas pela divisão de saúde mental da OMS (World Health Organization [WHO], 1996) e validadas no Brasil (Bandeira, Ishara e Zuardi, 2007). O questionário sociodemográfico e ocupacional conteve os seguintes dados: estado civil, sexo do profissional, tempo de vínculo com a instituição em anos completos, tempo de experiência na Psiquiatria em anos completos, carga horária de trabalho, turno de trabalho, percepção de segurança no ambiente de trabalho. O questionário SATIS-BR possui 32 questões que visam avaliar o grau de satisfação dos profissionais de saúde. Essas questões estão agrupados em quatro subescalas que avaliam: satisfação com a qualidade dos serviços prestados (alfa de Cronbach $=0,83$ ); satisfação da equipe em relação à sua participação no serviço (alfa de Cronbach $=0,72)$; satisfação em relação às condições gerais de trabalho (alfa de Cronbach $=0,77$ ); e satisfação em relação ao relacionamento interpessoal com os colegas de trabalho e superiores (alfa de Cronbach = $0,63)$. 
A escala global tem o objetivo de avaliar a satisfação geral da equipe com o serviço de saúde mental. Cada uma das 32 questões contêm respostas que se encontram dispostas em uma escala do tipo Likert com cinco pontos. Para avaliar o nível de satisfação global da equipe de enfermagem com o serviço de saúde mental e de cada subescala, é realizado o cálculo da média aritmética das respostas obtidas. A média das respostas varia de um a cinco, sendo que quanto mais próximo de cinco for o valor, maior o nível de satisfação do profissional (Bandeira et al., 2007).

A escala IMPACTO-BR possui 18 questões e três dimensões que visam avaliar os efeitos do trabalho sobre a saúde física e mental (cinco questões; alfa de Cronbach=0.78); os efeitos do trabalho sobre o funcionamento da equipe (seis questões; alfa de Cronbach=0.77); os efeitos do desenvolvimento das atividades sobre o estado emocional (cinco questões; alfa de Cronbach $=0.70$ ) e duas questões gerais que compõem a escala global (alfa de Cronbach=0.87). Cada uma das 18 questões contêm respostas que se encontram dispostas em uma escala do tipo Likert com cinco pontos. Para avaliar a sobrecarga global do trabalho e de cada subescala é realizado o cálculo da média aritmética das respostas obtidas. A média das respostas varia de um a cinco, sendo que quanto mais próximo de cinco for o valor, maior o nível de sobrecarga de trabalho sentida pelo profissional (Bandeira et al., 2007).

Os dados foram duplamente digitados em uma planilha de Excel, sendo analisados por meio do ambiente computacional e software estatístico R. A análise descritiva foi realizada sendo calculadas as frequências e percentuais para as variáveis categóricas; média, desviopadrão e percentis para as variáveis quantitativas. As variáveis quantitativas foram submetidas à análise de normalidade pelo teste de Shapiro-Wilk. O coeficiente de correlação de Spearman foi calculado para verificar a relação entre a escala SATIS-BR e IMPACTO-BR. Foi considerado o nível de significância de $5 \%$. Os valores missing não foram incluídos na análise.

Foram seguidos todos os preceitos éticos de acordo com a Resolução 466/2012, tendo aprovação pelo CEP com Parecer no 237.265. Os participantes assinaram o Termo de Consentimento Livre e Esclarecido.

\section{RESULTADOS}

Dos 255 profissionais de enfermagem, 240 atenderam aos critérios de inclusão.
Desses, 30 encontravam-se de licença médica, férias regulamentares ou férias prêmio, 25 se recusaram a participar do estudo e 82 não retornaram os questionários. No total, 103 profissionais responderam ao questionário, sendo 42 técnicos de um hospital e 61 de outro.

O tempo médio de experiência na psiquiatria foi de 5.9 anos com variação de 1 a 35 anos de experiência. A média do tempo de vínculo na instituição foi de 6.2 anos, sendo que $50 \%$ dos respondentes tiveram menos que 2 anos de vínculo. A maioria dos participantes era do sexo feminino (72.2\%), vivia com companheiro (61.9\%). Em relação ao turno de trabalho e carga horária semanal, houve predomínio de profissionais que trabalham no período diurno (53.6\%) e que possuem carga horária de 40 horas semanais $(55.1 \%)$. Ao serem perguntados sobre a percepção de segurança no ambiente de trabalho, a maioria (87.4\%) relatou não se sentir seguro para trabalhar conforme pode ser verificado na Tabela 1.

Tabela 1 - Características Sociodemográficas e Profissionais dos Técnicos de Enfermagem. $(\mathrm{n}=97)^{\star}$. Belo Horizonte, MG, Brasil, 2016

\begin{tabular}{|c|c|}
\hline Variáveis & n (\%) \\
\hline \multicolumn{2}{|l|}{ Sexo } \\
\hline Feminino & $70(72.2)$ \\
\hline Masculino & $27(27.8)$ \\
\hline \multicolumn{2}{|l|}{ Estado civil } \\
\hline Com companheiro & $60(61.9)$ \\
\hline Sem companheiro & $37(38.1)$ \\
\hline \multicolumn{2}{|l|}{ Turno de trabalho } \\
\hline Diurno & $52(53.6)$ \\
\hline Noturno & $45(46.4)$ \\
\hline \multicolumn{2}{|c|}{ Percepção de segurança $(n=95)$} \\
\hline Sim & $12(12.6)$ \\
\hline Não & $83(87.4)$ \\
\hline \multicolumn{2}{|c|}{ Carga horária - horas semanais } \\
\hline 20 & $2(2.0)$ \\
\hline 30 & $42(42.9)$ \\
\hline 40 & $54(55.1)$ \\
\hline \multicolumn{2}{|c|}{ Tempo de experiência na psiquiatria (anos) } \\
\hline Média (DP) & $5.9(7.8)$ \\
\hline p50 & 2.0 \\
\hline mín-máx & $1-35$ \\
\hline \multicolumn{2}{|c|}{ Tempo de vínculo na instituição (anos) } \\
\hline Média (DP) & $6.2(7.6)$ \\
\hline p50 & 2.0 \\
\hline mín-máx & $1-35$ \\
\hline
\end{tabular}

Seis profissionais não retornaram o questionário de identificação sociodemográfica e ocupacional. 
No que se refere à sobrecarga no trabalho avaliada pelo questionário IMPACTO-BR, observa-se que os profissionais apresentaram baixos escores para a escala geral e suas subescalas. A subescala "efeitos no funcionamento da equipe" apresentou os maiores escores demonstrando maior sobrecarga quando comparada às outras subescalas. Para essa subescala, é possível constatar que $25 \%$ dos profissionais apresentou índices de sobrecarga maior que 3.2 conforme pode ser verificado na Tabela 2. Os pontos que mais sobressaíram na sobrecarga dos profissionais foram: a possibilidade de ser agredido (3.9) e o cansaço físico ao final do trabalho (3.4). Por outro lado, as questões que apresentaram os menores índices de sobrecarga foram: a presença de distúrbios de sono relacionados ao trabalho (1.1), necessidade de procurar um psiquiatra em decorrência de problemas emocionais associados ao emprego (1.5), interferência do trabalho com portadores de transtorno mental sobre os relacionamentos familiares (1.5) e sobre a vida social (1.5).

Tabela 2 - Pontuações Obtidas para a Escala IMPACTO-BR entre Técnicos de Enfermagem. Belo Horizonte, MG, Brasil, $2016(n=103)$

\begin{tabular}{|l|c|c|c|c|c|}
\hline & $\begin{array}{c}\text { Média } \\
(\mathbf{D P})\end{array}$ & p25 & p50 & p75 & Mín-Máx \\
\hline Impacto global & $2.2(0.7)$ & 1.6 & 2.2 & 2.7 & $1.0-4.0$ \\
\hline $\begin{array}{l}\text { Efeitos sobre a saúde } \\
\text { física e mental }\end{array}$ & $2.0(0.8)$ & 1.2 & 1.9 & 2.6 & $1.0-3.8$ \\
\hline $\begin{array}{l}\text { Efeitos no funciona- } \\
\text { mento da equipe }\end{array}$ & $2.5(0.7)$ & 1.8 & 2.3 & 3.2 & $1.0-4.3$ \\
\hline $\begin{array}{l}\text { Repercussões emo- } \\
\text { cionais }\end{array}$ & $2.4(0.7)$ & 2.0 & 2.4 & 3.0 & $1.0-4.2$ \\
\hline
\end{tabular}

Com relação aos resultados obtidos através do questionário SATIS-BR, o escore de satisfação global com o trabalho foi de 3.1 (0.5) com variação de 1.9 a 4.8 pontos (Tabela 3). Grande número de participantes (42.7\%) demonstrou insatisfação por meio dos índices encontrados para o escore geral da escala SATIS-BR e suas quatro subescalas $(n=44)$. As subescalas que apresentaram maior e menor nível de satisfação foram respectivamente "satisfação com os relacionamentos no trabalho (3.4)" e "satisfação com as condições de trabalho (2.7)". Ao se considerar as questões avaliadas, os menores índices de satisfação estiveram relacionados à estrutura física (2.4), conforto (2.3), segurança (1.8) e salário (2.0). Já os maiores índices de satisfação estiveram ligados ao relacionamento com os colegas (3.9) e ao clima de amizade no ambiente de trabalho (3.8).
Tabela 3 - Pontuações Obtidas para a Escala SATIS-BR entre Técnicos de Enfermagem. Belo Horizonte, MG, Brasil, 2016 ( $\mathrm{n}=103)$

\begin{tabular}{|l|c|c|c|c|c|}
\hline & $\begin{array}{c}\text { Média } \\
(\mathbf{D P})\end{array}$ & p25 & p50 & p75 & Mín-Máx \\
\hline Satisfação geral & $3.1(0.5)$ & 2.6 & 3.1 & 3.4 & $1.9-4.8$ \\
\hline $\begin{array}{l}\text { Satisfação com a } \\
\text { qualidade dos ser- } \\
\text { viços }\end{array}$ & $3.2(0.6)$ & 2.7 & 3.4 & 3.6 & $1.4-5.0$ \\
\hline $\begin{array}{l}\text { Satisfação com a } \\
\text { participação }\end{array}$ & $2.8(0.7)$ & 2.4 & 2.8 & 3.2 & $1.3-5.0$ \\
\hline $\begin{array}{l}\text { Satisfação com as } \\
\text { condições de trabalho }\end{array}$ & $2.7(0.7)$ & 2.3 & 2.7 & 3.2 & $1.2-5.0$ \\
\hline $\begin{array}{l}\text { Satisfação com os } \\
\text { relacionamentos no } \\
\text { trabalho }\end{array}$ & $3.4(0.7)$ & 3.0 & 3.6 & 4.0 & $1.0-5.0$ \\
\hline
\end{tabular}

Pela análise do coeficiente de correlação de Spearman foi possível evidenciar a presença de correlação negativa entre os escores de satisfação e sobrecarga no trabalho. A subescala que avalia as repercussões emocionais apresentou associação estatística significante com todas as subescalas que avaliam a satisfação relacionada ao trabalho. Já em relação ao impacto sobre o funcionamento da equipe encontrou-se relação estatística significante com a satisfação em relação às condições de trabalho e com o escore geral da escala SATIS-BR (Tabela 4).

Tabela 4 - Coeficientes de Correlação de Spearman entre as Pontuações das Escalas SATIS-BR e IMPACTO-BR. Belo Horizonte, MG, Brasil, 2016 ( $n=103)$

\begin{tabular}{|l|l|l|l|l|}
\hline & $\begin{array}{l}\text { Impacto } \\
\text { global }\end{array}$ & $\begin{array}{l}\text { Saúde física } \\
\text { e mental }\end{array}$ & $\begin{array}{l}\text { Funciona- } \\
\text { mento da } \\
\text { equipe }\end{array}$ & $\begin{array}{l}\text { Reper- } \\
\text { cussões } \\
\text { emocionais }\end{array}$ \\
\hline Satisfação geral & $-0.28^{* * *}$ & -0.16 & $-0.20^{* *}$ & $-0.36^{* * *}$ \\
\hline $\begin{array}{l}\text { Satisfação com } \\
\text { a qualidade dos } \\
\text { serviços }\end{array}$ & $-0.25^{* * *}$ & -0.19 & -0.16 & $-0.29^{* * *}$ \\
\hline $\begin{array}{l}\text { Satisfação com a } \\
\text { participação }\end{array}$ & $-0.20^{* *}$ & -0.12 & -0.13 & $-0.27^{* * *}$ \\
\hline $\begin{array}{l}\text { Satisfação com } \\
\text { as condições de } \\
\text { trabalho }\end{array}$ & $-0.33^{* * *}$ & -0.19 & $-0.30^{* * *}$ & $-0.37^{* * *}$ \\
\hline $\begin{array}{l}\text { Satisfação com } \\
\text { os relacionamen- } \\
\text { tos no trabalho }\end{array}$ & -0.13 & -0.07 & -0.01 & $-0.19^{* *}$ \\
\hline
\end{tabular}

** Significante ao nível de confiança de 95\%

*** Significante ao nível de confiança de $99 \%$ 


\section{DISCUSSÃO}

Os técnicos de enfermagem que participaram deste estudo demonstraram que as atividades desenvolvidas nas instituições psiquiátricas onde trabalham acarretam, em sua maioria, baixos níveis de sobrecarga de trabalho, todavia, o maior nível de sobrecarga sentido foi sobre o funcionamento da equipe. Ainda assim, os profissionais participantes desta pesquisa apresentaram maiores níveis de sobrecarga relacionada ao trabalho quando comparados aos resultados encontrados entre profissionais de outro hospital psiquiátrico no Brasil (Alves et al., 2013).

No que se refere à sobrecarga sentida em relação ao funcionamento da equipe, constatou-se que os maiores níveis de sobrecarga estiveram relacionados à percepção de insegurança dentro do ambiente de trabalho e ao cansaço físico e mental sentido após os plantões. De forma semelhante, a questão da segurança no ambiente hospitalar da psiquiatria foi associada ao desenvolvimento de maiores níveis de stress demonstrando também que as condições do ambiente de trabalho e o cuidado ao portador de transtorno mental acarretam maior sobrecarga psíquica do que física (Qi et al., 2014). Em outra pesquisa realizada na Bélgica foi possível verificar que a sobrecarga no trabalho se relaciona diretamente ao desenvolvimento de exaustão emocional e burnout influenciando indiretamente a qualidade do cuidado (Boagaert et al., 2013).

Por outro lado, os níveis de satisfação demonstrados pelos técnicos de enfermagem apresentaram maior variação conforme pode ser visto no escore de satisfação global, sendo possível verificar que uma porcentagem significativa dos participantes se encontrava insatisfeita com seu emprego. Em relação aos baixos níveis de satisfação pode-se observar que as condições de trabalho relacionadas aos aspectos de estrutura física, conforto, segurança e salário alcançaram os menores índices de satisfação. É importante ressaltar que estas duas instituições apresentam estrutura antiga e nunca passaram por reformas estruturais maiores, reproduzindo a estrutura física existente anterior à reforma psiquiátrica. Da mesma maneira que encontrado nesta pesquisa, um estudo realizado em um Centro de Atenção Psicossocial (CAP) com 23 profissionais de saúde também constatou baixos níveis de satisfação relacionados às condições de trabalho, sugerindo a presença de um problema estrutural nas redes de atenção em saúde mental (Ferreira, 2015).
No entanto, outras pesquisas avaliativas precisam ser realizadas, uma vez que estudos que investiguem a qualidade dos serviços de psiquiatria sob essa perspectiva ainda são escassos.

Pelo exposto, é possível afirmar que as condições de trabalho se relacionam tanto com os níveis de satisfação e a sobrecarga de trabalho entre os profissionais de enfermagem sendo fatores reconhecidamente importantes nos processos de adoecimento (Leka, Hassard \& Yanagida, 2012; Gandhi et al., 2014). Condições de trabalho precárias vivenciadas por enfermeiros e técnicos de enfermagem podem ser constatadas ao se verificar os baixos salários e exposição contínua a riscos ocupacionais responsáveis pelo adoecimento, altas taxas de absenteísmo e diminuição da capacidade para o trabalho (Felli, 2012; Wisniewski, Silva, Évora e Matsuda, 2015). Soma-se a isso a deficiência de recursos humanos e materiais presentes em instituições públicas, o que interfere na qualidade dos serviços e na satisfação dos profissionais que trabalham prestando assistência à população (Alves et al., 2013).

Conforme pode ser verificado na presente pesquisa, a satisfação dos técnicos de enfermagem encontrou-se inversamente relacionada à sobrecarga emocional no trabalho o que chama a atenção para a necessidade de estabelecer estratégias que favoreçam a proteção física e psíquica desses trabalhadores. As desordens psicológicas representam a segunda causa de afastamento entre profissionais de enfermagem, especialmente entre técnicos de enfermagem (Santos, Mamede e Paula, 2014), sendo elas responsáveis pelo maior número de dias perdidos por problemas de saúde (Felli, 2012).

Adicionalmente, é importante mencionar que os técnicos de enfermagem apresentaram baixos índices de satisfação em relação às oportunidades de participação no serviço. Essa dimensão avalia a satisfação em relação ao nível de responsabilidade que esses profissionais possuem, oportunidades de promoção, participação nas decisões e aceitação das opiniões expressas no ambiente de trabalho (Ferreira, 2015).

De acordo com a teoria de Herzberg, os fatores determinantes da satisfação no trabalho podem ser classificados como extrínsecos e intrínsecos. Os primeiros dizem respeito ao retorno financeiro e as condições de trabalho relacionadas à estrutura física e ambiente. Para Herzberg os fatores extrínsecos determinam o nível de satisfação relacionado ao trabalho, porém, não podem determinar o nível de motivação. 
Os fatores intrínsecos relacionados à motivação estão interligados com o nível de autonomia, participação, reconhecimento profissional e responsabilidade (Herzberg, Mausner \& Snyderman, 1959).

Os resultados encontrados em relação às oportunidades de participação dos profissionais de enfermagem no ambiente de trabalho estão em consonância com os resultados da pesquisa realizada por Leal et al. (2012) reforçando a presença de estruturas hierarquizadas que pouco contribuem para o desenvolvimento de trabalho em equipes interdisciplinares, condição sine qua non para serviços de saúde mental. Essas estruturas impedem a participação dos profissionais e a troca de conhecimentos, importantes no processo de decisão terapêutica e na manutenção da continuidade do cuidado (Leal et al., 2012).

Este estudo apresenta algumas limitações relativas à natureza do estudo transversal que não permitem demonstrar as relações causais existentes. Adicionalmente, as escalas utilizadas foram elaboradas para ser autoaplicáveis, ficando sujeito à confiabilidade das respostas fornecidas pelos profissionais que da pesquisa. Os achados deste estudo são baseados em uma amostra autosselecionada e todos os resultados e interpretações são limitados a esse grupo de respondentes. No entanto, destaca-se que se obteve uma taxa de resposta que correspondeu a grande parte técnicos de enfermagem dos dois hospitais. Além disso, é importante mencionar que esta pesquisa foi realizada em dois dos quatro hospitais localizados no município.

\section{CONCLUSÃO}

Os resultados permitiram identificar que os técnicos de enfermagem que participaram deste estudo apresentaram baixos níveis de sobrecarga relacionados ao trabalho, sendo o maior nível de sobrecarga associado ao funcionamento da equipe. Em relação à satisfação, uma porcentagem significativa dos participantes demonstrou-se insatisfeita, sobretudo no que diz respeito às condições de trabalho e à possibilidade de participar das decisões e projetos desenvolvidos nas duas instituições. Também foi possível verificar a presença de relação inversamente proporcional entre grande parte das dimensões da escala de satisfação e as dimensões da escala que avalia a sobrecarga existente no trabalho.
Destaca-se a necessidade de considerar outros aspectos na avaliação da qualidade dos serviços de saúde mental oferecidos à população, bem como de repensar as condições de trabalho vivenciadas pelos profissionais de enfermagem. Pesquisas adicionais devem ser realizadas a fim de investigar as consequências e relações existentes para os níveis de satisfação e sobrecarga relacionados ao trabalho e as condições de saúde desses profissionais.

\section{REFERÊNCIAS BIBLIOGRÁFICAS}

Alves, A. P., Guidetti, G. E. C. B., Diniz, M. A., Rezende, M. P., Ferreira, L. A., e Zuffi F. B. (2013). Avaliação do impacto do trabalho em profissionais de saúde mental de uma instituição psiquiátrica. Revista Mineira de Enfermagem, 17(2), 424-428.

Bandeira, M., Ishara, S., e Zuardi, A. W. (2007). Satisfação e sobrecarga de profissionais de saúde mental: Validade de construto das escalas SATIS-BR e IMPACTOBR. Jornal Brasileiro de Psiquiatria, 56(4), 280-286.

Bogaert, P. V., Clarke, S., Wouters, K., Franck, E., Willems, R., \& Mondelaers, M. (2013). Impacts of unit-level nurse practice environment, workload and burnout on nurse-reported outcomes in psychiatric hospitals: A multilevel modelling approach. International Journal of Nursing Studies, 50, 357-365.

Felli, V. E. A. (2012). Condições de trabalho de enfermagem e adoecimento: Motivos para a redução da jornada de trabalho para 30 horas. Enfermagem em Foco, 3(4), 178-181.

Ferreira, A. P. (2015). Satisfação, sobrecarga de trabalho e estresse nos profissionais de serviço de saúde mental. Revista Brasileira de Medicina do Trabalho, 13(2), 9199.

Gandhi, S., Sangeetha, G., Ahmed, N., \& Chaturvedi, S. K. (2014). Somatic symptoms, perceived stress and perceived job satisfaction among nurses working in an Indian psychiatric hospital. Asian Journal of Psychiatry, $12,77-81$. 
Herzberg, F., Mausner, B., \& Snyderman, B. (1959). The motivation to work (2nd ed.). New York: Wiley.

Hamaideh, S. H. (2011). Burnout, social support and job satisfaction among Jordanian mental health nurses. Issues in Mental Health Nursing, 32(4), 234-242.

Leal, R. M. A. C., Bandeira, M. B., e Azevedo, K. R. N. (2012). Avaliação da qualidade de um serviço de saúde mental na perspectiva do trabalhador: Satisfação, sobrecarga e condições de trabalho dos profissionais. Psicologia: Teoria e Prática, 14(1), 15-25.

Leka, S., Hassard, J., \& Yanagida, A. (2012). Investigating the impact of psychosocial risks and occupational stress on psychiatric hospital nurses' mental well-being in Japan. Journal of Psychiatric and Mental Health Nursing, 19, 123-131.

Organização Mundial da Saúde. (2001). Relatório Mundial da Saúde. Saúde mental: Nova concepção, nova esperança. Lisboa: Divisão de Saúde Mental da Organização Mundial da Saúde.
Qi, Y., Xiang, Y., An, F., Wang, J., Zeng, J., Ungvari, G. S., ...\& Chiu, H. F. K. (2014). Nurses' work-related stress in China: A comparison between psychiatric and general hospitals. Perspectives in Psychiatric Care, 50(1), 27-32.

Rebouças, D., Legay, L. F., e Abelha, L. (2007). Satisfação com o trabalho e impacto causado nos profissionais de serviço de saúde mental. Revista de Saúde Pública, 41(2), 244-250.

Santos, N. A. C., Mamede, N. M., e Paula, M. A. B. (2014). Principais causas de afastamento do trabalho na equipe de enfermagem: Revisão integrativa da literatura. Revista de Administração em Saúde, 16(64), 97-103.

World Health Organization. WHO-SATIS (1996). Consumer's and caregivers' satisfaction with mental health services: A multisite study. WHO Division of Mental Health.

Wisniewski, D., Silva, E. S., Évora, Y. D. M., e Matsuda, L. M. (2015). Satisfação profissional da equipe de enfermagem X condições e relações de trabalho: Estudo relacional. Texto e Contexto Enfermagem, 24(3), 850-858.

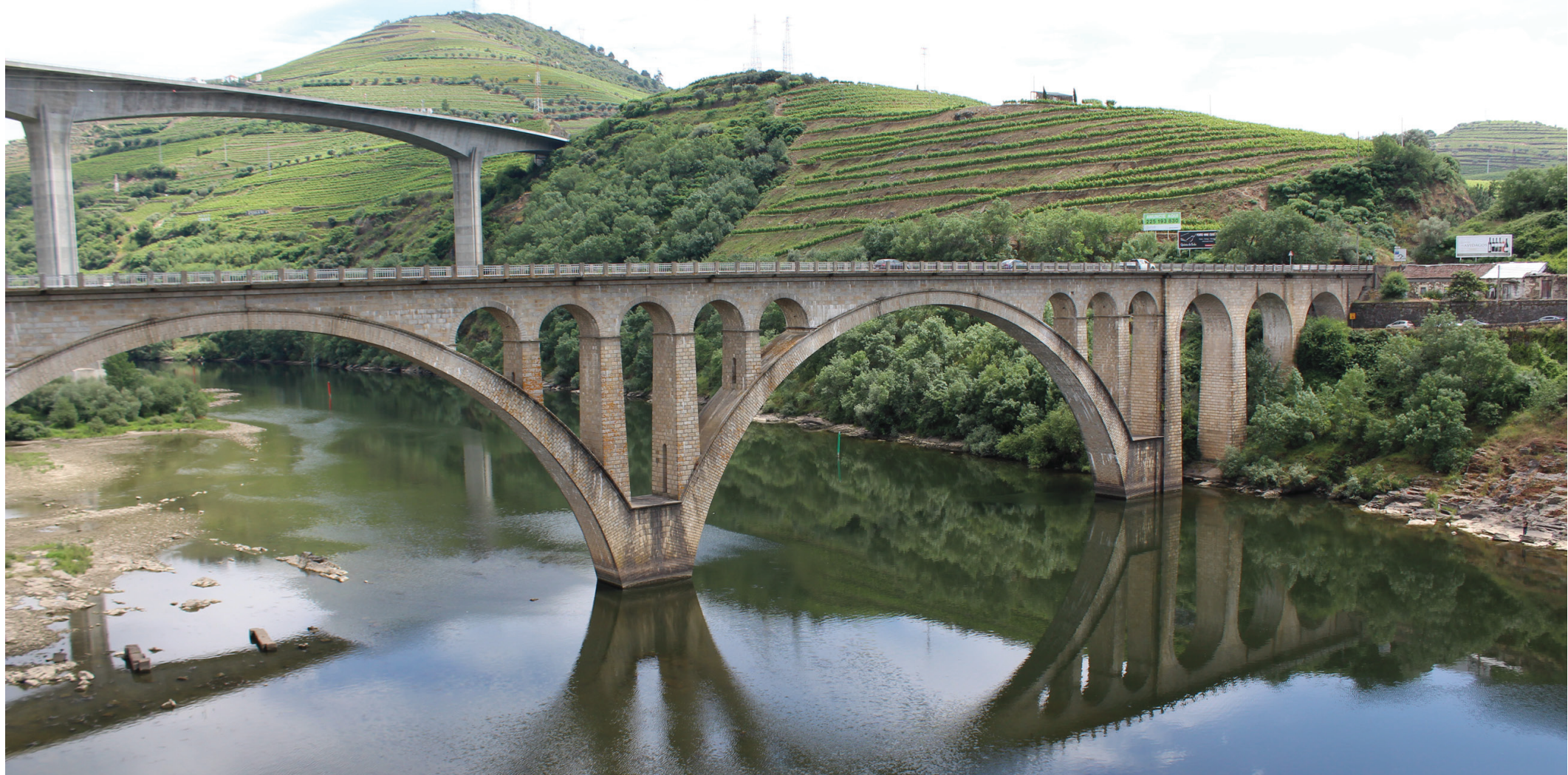

